\title{
El Poder Persuasivo de la Refutación en Argumentaciones Colectivas
}

\section{The Persuasive Power of Refutation in Collective Argumentation}

\author{
Jonathan Cervantes-Barraza* \\ Guadalupe Cabañas-Sánchez ** \\ Joan Sebastian Ordoñez-Cuastumal ${ }^{* * *}$
}

\begin{abstract}
Resumen
Este estudio está enmarcado en la argumentación matemática en el salón de clases. Tuvo como propósito identificar funciones que cumple la refutación de aserciones en argumentaciones colectivas. Esto se llevó a cabo mediante la implementación de un enfoque teórico metodológico fundamentado en el modelo argumentativo de Toulmin (2003), el cual permitió el estudio de las argumentaciones colectivas y sus estructuras dentro del salón de clases. Conjunto con lo establecido en la teoría se encontró cómo la refutación tiene un poder persuasivo sobre los argumentos de los estudiantes, ésta otra función de la refutación.
\end{abstract}

Palabras clave: Refutaciones. Argumentaciones colectivas. Persuasión y Modelo de Toulmin.

\begin{abstract}
This study is framed in mathematical classroom argumentation. Its purpose was to identify functions of refutation of claims in collective argumentation. This was done through the implementation of a theoretical methodological approach based on the argumentative model of Toulmin (2003), which allowed the study of the collective argumentation and their structures within the classroom. Along with the theory convictions, we found how refutation has a persuasive power on the student's arguments, with this being another function of refutation.
\end{abstract}

Keywords: Refutations. Collective argumentation. Persuasion and Model of Toulmin.

\section{Introducción}

En este artículo se presenta una investigación centrada en el estudio de la refutación y la reconstrucción de argumentos en clase de matemáticas, empleando el modelo

\footnotetext{
* Maestro en Ciencias-Área Matemática Educativa, Universidad Autónoma de Guerrero (UAGro), Guerrero, México. Dirección Postal: Av. Lázaro Cárdenas S/N, Ciudad Universitaria Chilpancingo, CP 39087, Guerrero, México. E-mail: jacbmath@hotmail.com

** Doctora en Ciencias con Especialidad Matemática Educativa por el Departamento de Matemática Educativa del Centro de Investigación y Estudios Avanzados del IPN (CIEA/IPN). Profesora e investigadora de la Universidad Autónoma de Guerrero (UAGro), Guerrero, México. Dirección Postal: Av. Lázaro Cárdenas S/N, Ciudad Universitaria Chilpancingo, CP 39087, Guerrero, México. E-mail: gcabanas.sanchez@gmail.com

*** Maestro en Ciencias-Área Matemática Educativa, Universidad Autónoma de Guerrero (UAGro), Guerrero, México. Dirección Postal: Av. Lázaro Cárdenas S/N, Ciudad Universitaria Chilpancingo, CP 39087, Guerrero, México. E-mail: joseor910831@gmail.com
} 
argumentativo de Toulmin (2003). Así mismo, enfocada en estudiar el papel que desempeña uno de los elementos que no se tiene en cuenta; la refutación, en el contexto de resolución de una actividad matemática (INGLIS; MEJÍA-RAMOS; SIMPSON, 2007, p.6).

Una actividad es un universo de posibles operaciones que un individuo puede o no tomar mientras trabaja en un problema, a través de un producto certero, con recursos certeros, la retroalimentación que el problema puede proveer en esas operaciones y las operaciones adaptadas a la retroalimentación (HERBST, 2006). Permitiendo que los estudiantes, a través de la actividad, experimenten un proceso de argumentación relacionado con un conocimiento matemático específico. Luego de que los estudiantes realizaron la actividad, se procedió a esquematizar los argumentos con el modelo argumentativo de Toulmin y analizar las refutaciones establecidas por los estudiantes. Así, el propósito de esta investigación es identificar las funciones de la refutación durante la argumentación colectiva entre estudiantes de básica secundaría.

\section{La pregunta problema y su contexto}

En investigaciones previas se manifiesta la importancia que prevé el análisis de la reconstrucción y evaluación de argumentos en matemáticas, convirtiéndose en una línea de investigación importante en la Educación Matemática (INGLIS; MEJÍA-RAMOS, 2005). Así también, investigadores como (INGLIS; MEJÍA-RAMOS, 2005, 2009; INGLIS; MEJÍARAMOS; SIMPSON, 2007) argumentaron que al implementar el modelo completo de Toulmin es posible modelar fielmente todos los aspectos de la argumentación. Por consecuente, estas investigaciones evidencian la utilidad del modelo completo de Toulmin para estructurar argumentos matemáticos en un sentido lógico y valedero.

Por otro lado, investigaciones han usado el modelo argumentativo de Toulmin (2003), con la tendencia de ignorar el rol del calificador modal y la refutación, partes sustanciales de la argumentación (YACKEL, 2002; WHITENACK; KNIPPING, 2002; KRUMMHEUER, 1995, 2007, 2013) implementando el modelo reducido o núcleo (e. i., evidencia, garantía y aserción) para reconstruir las estructuras argumentativas de los estudiantes de primaria y analizarlas, pero sin tener en cuenta partes del argumento como el calificador modal y la refutación.

De todas estas investigaciones realizadas en el campo de la argumentación, hay una preocupación seria que concierne el análisis de los argumentos desde una perspectiva que no tiene en cuenta la diversidad de calificadores modales y refutaciones utilizados en las 
argumentaciones matemáticas (INGLIS; MEJÍA-RAMOS, 2005). Por consecuente, la oportunidad de refutar el proceso argumentativo de los estudiantes se promueve con el propósito de ayudarlos en su proceso de aprendizaje y en la producción de argumentos matemáticos. Estas oportunidades deberían estar presentes desde los primeros años de escolaridad y no reservadas para grados de estudios superiores (NCTM, 2000). Así, hacer caso omiso a las refutaciones en la argumentación matemática puede ocultar aspectos importantes en los argumentos de los estudiantes (INGLIS; MEJÍA-RAMOS, 2005).

En el mismo sentido, Giannakoulias et al. (2010) estudiaron cómo los profesores refutan las aserciones de un grupo de estudiantes en actividades matemáticas. Para ello, se fundamentaron en conceptos de refutación y contraejemplos en el sentido de Lakatos (1976), e implementaron el modelo argumentativo planteado por Toulmin (2003). En sus resultados plasmaron las formas en la cual los profesores refutan aserciones de estudiantes, éstas mediante la implementación de la teoría y/o el uso de contraejemplos.

Por su parte Yopp (2015) reporta el papel crucial de la refutación en las actividades propuestas a los docentes con aserciones falsas, las que se diseñaron con la intención de estudiar los tipos de aserciones y refutaciones establecidas por los docentes, basándose desde las respuestas dadas por los estudiantes en la clase de matemáticas. A modo de resultado, identificaron cómo los docentes establecieron conjeturas basándose en ideas matemáticas como soporte de las aserciones, refutaciones o diferentes razonamientos lógicos.

Otro sentido de abordar el estudio de la refutación es centrándose en el estudiante (FUJITA et al., 2011). Los autores emplearon la refutación, en el sentido de la prueba en geometría, con estudiantes de secundaria, con el objetivo de que éstos establecieran conjeturas basadas en razonamientos, debido a que los estudiantes realizaban hipótesis primitivas basadas en argumentos visuales. De igual forma, la refutación en la investigación llevada a cabo por Balacheff (1991) relaciona los contraejemplos y el conocimiento matemático en la lógica formal. La refutación, uno de los medios por el cual se indagó cómo establecían conjeturas y contraejemplos, con el objetivo de generar conocimiento desde diálogos matemáticos.

Reid, Knipping y Crosby (2011) evidenciaron la lógica de las prácticas del profesor cuando los estudiantes refutan los argumentos. Además, señalan las formas en las que un argumento puede ser refutado, y dan a conocer las partes del argumento que se invalidan o presentan una suficiencia adecuada. En el mismo sentido, Reid (2002) expone una de las funciones que cumple la refutación de conjeturas en un salón de clases de primaria. Siendo la refutación un medio por el cual se evidenció los razonamientos empleados por los estudiantes 
y cómo se presentan éstos en un contexto escolar. Luego de evidenciar la problemática y las investigaciones previas, referentes a la refutación en clase de matemáticas, se plantea la siguiente pregunta de investigación: ¿Qué otras funciones cumple la refutación de aserciones en la argumentación colectiva?

\section{Fundamentación teórica}

Con el objetivo de tener referentes que apoyen esta teóricamente está investigación, algunos conceptos se tomaron de los aportes realizados por Toulmin y otros en el campo de la argumentación matemática (TOULMIN, 2003).

\subsection{Argumentación}

Tomando el aporte de Goizueta y Planas (2013), quienes afirman que un argumento es una razón, o razones, ofrecidas en favor o en contra de una aserción, mientras que la argumentación es el acto de producir razones. Se constata que argumentación y argumentos no se refieren a lo mismo; argumentación es toda la actividad que se realiza en el acto de convencer a una audiencia, y argumento es una razón o series de razones ofrecidas por el argumentador. Sin embargo, esta investigación está ubicada en el campo de la Educación Matemática, por esta razón es importante saber qué se entiende por un argumento matemático. Whitenack y Knipping (2002) lo definen como el resultado de una argumentación llevada a cabo en una clase de matemáticas.

Por consecuente, se entiende el término argumentación para referirse a toda la actividad de hacer aserciones, refutándolas, soportándolas mediante la producción de razones y criticando esas razones. De igual forma, un argumento es una cadena de razonamiento, en términos de una secuencia conectada de aserciones y razones, que entre ellas establecen el contenido y la fuerza de la posición por la cual una persona argumenta (TOULMIN, 2003).

\subsection{Argumentaciones colectivas}

En el campo de la Educación Matemática se ha estudiado argumentación a través del modelo de Toulmin (2003) para investigar sobre: la estructura de los argumentos construidos por los profesores (e. g., GIANNAKOULIAS et al., 2010), estudiantes trabajando de forma 
colectiva (e. g., YACKEL, 2002; WHITENACK; KNIPPING, 2002; KRUMMHEUER, 1995, 2007) y por matemáticos (e. g., HAREL, 2007; INGLIS; MEJÍA-RAMOS; SIMPSON, 2007).

Tal como se presentó anteriormente, el modelo de Toulmin juega un papel importante en estudios relacionados con la argumentación colectiva (WHITENACK; KNIPPING, 2002; KRUMMHEUER, 2015; REID; KNIPPING; CROSBY, 2011). Estos autores implementaron el modelo de Toulmin como una herramienta metodológica que facilitó el análisis de la naturaleza de la actividad y dialogo en clase de matemáticas. Sin embargo, la argumentación colectiva establecida en Krummheuer (1995) y Conner et al., (2014), en términos generales, como varias personas que trabajan en conjunto para establecer una aserción, en el mismo sentido, la argumentación colectiva puede ser también enmarcada como una acción donde participan grupos de personas en los debates de una manera matemática (CONNER et al., 2014).

\subsection{Refutación en argumentaciones colectivas}

Para introducir el concepto de refutación se expone, de forma breve, la definición en el sentido de Toulmin (2003), una refutación es local a un paso de un argumento y especifica excepciones a la aserción/conclusión. Esto significa que la refutación muestra los casos donde la aserción o conclusión no es soportada por su garantía. Además, Walton (2009) estableció que una refutación tiene como objetivo mostrar que el argumento que se dirige en contra es cuestionable, en otras palabras es una especie de réplica que muestra que el argumento es insostenible.

De la misma manera Reid, Knipping y Crosby (2011), y Reid (2002) reconocen las formas en que un argumento puede implicar una refutación: los datos del argumento pueden ser refutados, dejando la conclusión en duda. Continuando con la garantía del argumento, la cual puede ser refutada dejando de nuevo la conclusión en duda o la conclusión en sí misma puede ser refutada, lo que implica que, o bien los datos o la garantía, no son válidas, pero no aclaró cuál.

\subsection{Modelo argumentativo de Toulmin}

En esta sección se expone uno de los sustentos teóricos sobre el cual se fundamenta la presente investigación, el modelo argumentativo de Toulmin (2003). Este modelo propuesto por Toulmin es útil para esquematizar los argumentos expuestos por los estudiantes en 
estructuras semánticas, además de usarlo para analizar y comparar la organización de los argumentos entre las fases de crear y explorar las cadenas de razonamientos (e .g., deductivos, inductivo, por analogías y abducción). Este modelo tiene una estructura constituida por seis elementos: aserción, garantía, respaldo, evidencia, calificador modal y refutación (Ver figura 1).



Figura 1-Estructura argumentativa de Toulmin Fuente: Toulmin (2003)

La aserción es la tesis que sustenta el argumentador, la evidencia $(E)$ es la información sobre la cual se fundamenta la aserción; la garantía $(W)$ tiene el papel de justificar la conexión entre la evidencia y la aserción. La garantía cuenta con un respaldo $(R e)$ que presenta una justificación general; el calificador modal $(C)$ especifica la fuerza de la aserción; tales como certeramente, presumiblemente, probablemente, siempre y otros, expresando el grado de confianza en la tesis. La refutación $(R)$ es caracterizada por presentar las excepciones de la aserción, aquellas condiciones bajo las cuales no se puede sostener la tesis del argumento (TOULMIN; RIKIE; JANIK, 1984).

\subsection{Argumentos formales e informales}

Visto anteriormente en el modelo argumentativo de Toulmin, la garantía brinda la conexión entre la evidencia y la aserción, presentando soporte a la aserción o conclusión del argumentador. De este modo, la garantía cumple un rol importante en la estructura argumentativa, este elemento da origen a la clasificación de argumentos; ya sean formales o informales. Así, según Viholainen (2008) un argumento es formal si su garantía es puramente basada en los elementos del sistema axiomático formal de las matemáticas. En otras palabras, un argumento es formal si explícitamente muestra cómo la aserción sigue lógicamente las definiciones, axiomas y, posteriormente, teoremas probados desde la información dada.

Por otra parte, un argumento es informal si sus garantías son basadas en el uso de aserciones informales de conceptos o situaciones en las cuales un argumento está relacionado. En el mismo orden de ideas, por interpretaciones informales, en este estudio se entienden 
como interpretaciones físicas o visuales realizadas por los estudiantes frente actividades matemáticas propuestas, tales como fundamentarse en la representaciones graficas sin presentar ninguna garantía sustentada en propiedades, teoremas, fórmulas matemáticas (VIHOLAINEN, 2008).

\section{Implementación metodológica}

\subsection{Participantes y diseño de la actividad}

Participaron en esta investigación veinte estudiantes (entre 11-12 años de edad) pertenecientes a un colegio del norte de Barranquilla, Atlántico (Colombia) inscritos en sexto año del nivel básico-secundaria. El papel del investigador fue participar de forma activa durante la aplicación de la actividad dentro del salón de clases y orientar a los estudiantes en la resolución de la tarea y propiciar la refutación de aserciones.

El desarrollo de la actividad consistió en dos momentos. Primero, los estudiantes resolvieron de forma individual $\mathrm{y}$, posteriormente, el profesor $^{1}$ dio lugar para que los estudiantes comentaran sus respuestas en forma colectiva, con el propósito de generar argumentación y se diera oportunidades a refutaciones de las aserciones dadas por los estudiantes. Durante la aplicación de la actividad, la interacción entre estudiantes y profesor fue videograbada, la clase tuvo una duración de una hora reloj y los estudiantes implementaron material escolar como: marcadores y las actividades impresas.

La actividad que se implementó en la investigación fue validada con dos estudiantes de diferentes colegios pertenecientes al mismo nivel escolar (Figura 2).

\footnotetext{
${ }^{1}$ El profesor quien aplicó la actividad es el primer autor de este artículo.
} 
En la cuadrícula siguiente se han construido los triángulos de color rojo, verde y azul. La cuadrícula está formada por cuadrados de $1 \mathrm{~cm}$ de lado, como se muestra en la figura.

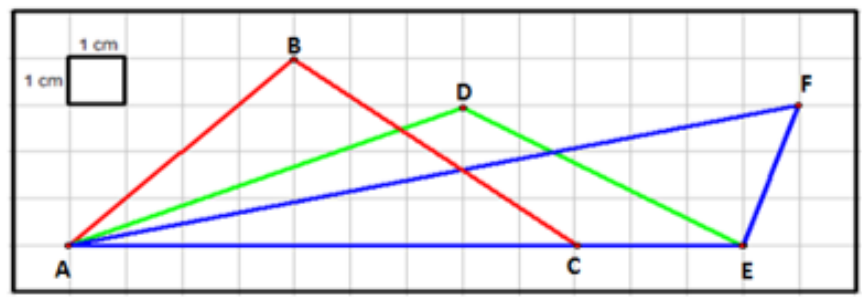

Analiza y responde: ¿qué triángulo tiene mayor área?, argumenta tu respuesta seleccionada.

A) El área del triángulo rojo $(\triangle \mathrm{ABC})$.

B) El área del triángulo verde ( $\triangle \mathrm{ADE})$.

C) El área del triángulo azul ( $\triangle \mathrm{AFE})$.

D) Ninguno, todos tienen la misma área.

Figura 2 - Actividad matemática propuesta. Fuente: autores

\subsection{Análisis de las escenas}

Para llevar a cabo el análisis de la escena presentada, se realizó la transcripción del video en una tabla (ver anexo) que contiene: el número de la escena, nombre del hablante y dialogo. El análisis se fundamentó en los argumentos de los estudiantes reconstruidos con el modelo de Toulmin (2003), así mismo, se identificaron los elementos del núcleo de un argumento (evidencia, aserción, garantía) sustentado en investigaciones (e. g. WAGNER et al., 2014; KRUMMHEUER, 1995, 2007; WHITENACK; KNIPPING, 2002). Aparte de identificar el núcleo de los argumentos, se identificó la refutación de los estudiantes durante la clase y se agregó en la estructura argumentativa.

Seguidamente, los conceptos de la teoría argumentativa se contrastaron con los resultados obtenidos para identificar aspectos sobresalientes en la evidencia recolectada. Así mismo, la clasificación de los argumentos según sus garantías como lo expone (VIHOLAINEN, 2008) en argumentos formales o informales.

\subsection{Convenciones del modelo de Toulmin}

En esta investigación el análisis de la argumentación se llevó a cabo mediante la implementación del modelo argumentativo propuesto por Toulmin (2003). Sobre éste se realizó una serie de convenciones que facilita el análisis de la argumentación. En los argumentos se identificaron: evidencias, garantías, aserciones, y refutación (e. g., 
KRUMMHEUER， 1995，2007，2015; WHITENACK; KNIPPING，2002; BIKNERAHSBAHS; KNIPPING; 2015, REID; KNIPPING; CROSBY, 2011).

De igual manera, las convenciones realizadas en el modelo argumentativo de Toulmin (ver Figura 3) tienen que ver con representar: la Evidencia con un rectángulo, la Garantía con un ovalo, la Aserción con un rectángulo con esquinas curvas y, por último, la Refutación con un rombo. Dentro de cada figura geométrica se encuentra señalado el nombre del participante con negritas, y el texto transcrito del video.

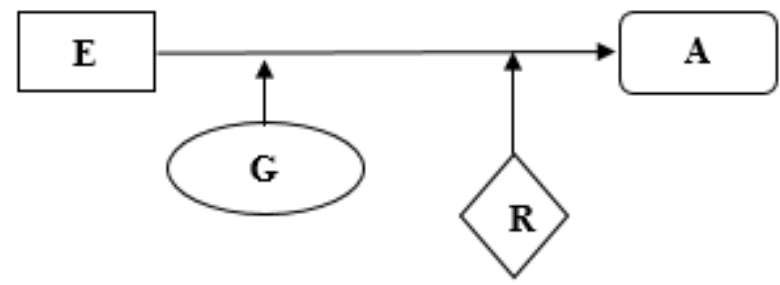

Figura 3 - Modelo argumentativo y sus convenciones Fuente: Autores

\section{Resultados}

En esta sección se presentan los resultados de la investigación en términos de estructuras que representan los argumentos reconstruidos según lo descrito en la sección de metodología. Estos argumentos conforman el cuerpo completo de la argumentación colectiva llevada a cabo en la clase. Primeramente, se presentan de forma individual y, finalmente, se presenta la estructura de todos los argumentos colectivos.

\subsection{Argumentos persuadidos por la refutación}

Con base en la actividad propuesta, los estudiantes dieron a conocer sus respuestas: la primera respuesta fue por parte del estudiante Nicolás, quien realizó la aserción el área del triángulo verde es el que tiene mayor área (Figura 1.) 


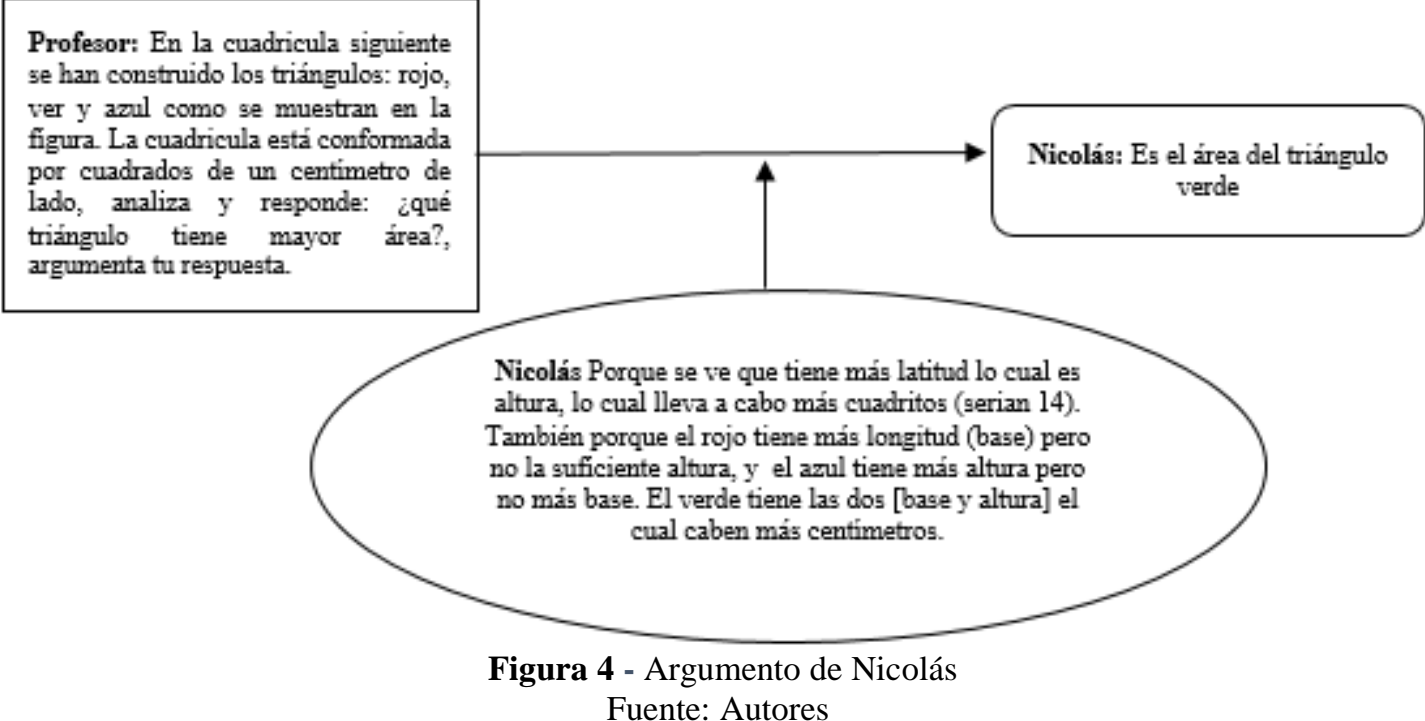

En el argumento presentado por Nicolás, la garantía se fundamentó en características del triángulo (i.e., medidas de la altura, base y número de cuadros) desde lo visual. Además, el estudiante realizó una comparación entre las medidas de la base y altura de los demás triángulos con el propósito de estimar una medida del área para cada uno, sin mencionar la relación existente entre éstas. Cabe resaltar que la garantía de este argumento se apoya desde rasgos visuales y no implementó un sustento matemático alguno, por consiguiente el argumento de Nicolás es un argumento de tipo informal.

Posteriormente de la presentación del argumento por parte de Nicolás, el estudiante Ángel respondió: Yo creo que el que tiene más área es el azul [triángulo azul]. Esta respuesta es la aserción en relación con la actividad propuesta. Así mismo, expuso su garantía basada en la cantidad de cuadros que contiene el triángulo, éste tiene mayor área que los demás triángulos, y soporta el hecho de que el triángulo azul tenga mayor área. Cabe resaltar que el argumento expuesto por este estudiante es de tipo informal, ya que no implementa formulas o teoremas matemáticos que sustenten el porqué de su aserción es verdadera (Figura 5).

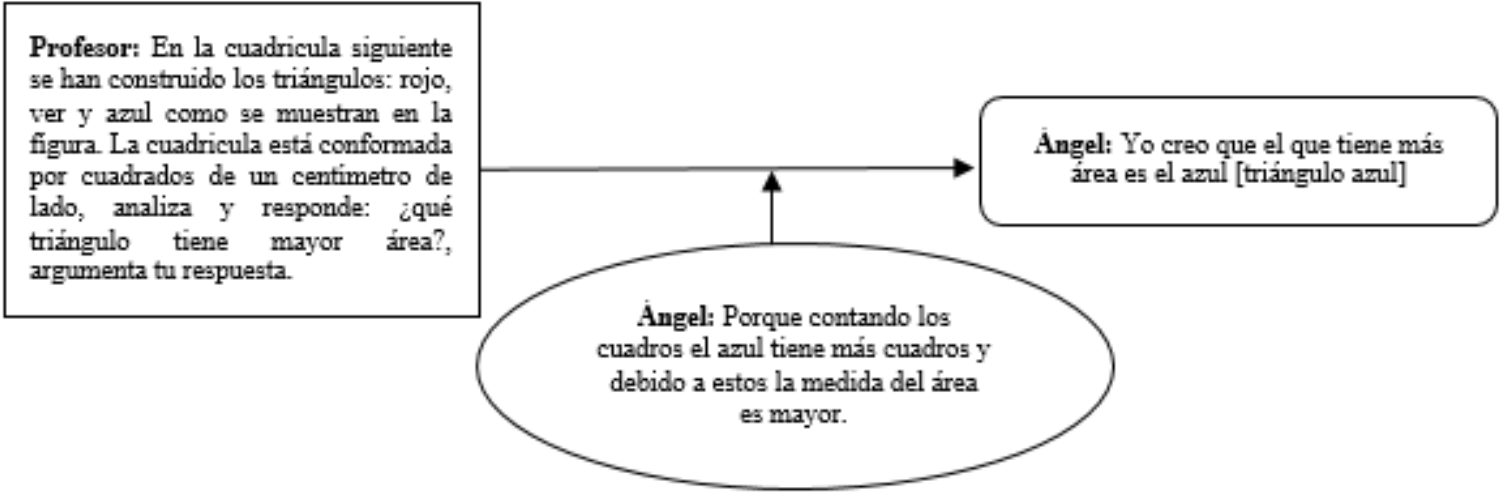

Figura 5 - Argumento de Ángel Fuente: Autores 
Consecutivamente, el estudiante Guido estableció su aserción, el triángulo azúl debido a que tiene más cuadros y presenta su garantía Como cada cuadro da $2 \mathrm{~cm}$, medio cuadro es $1 \mathrm{~cm}$. La garantía es de tipo informal y fundamenta su argumento en el área de cada cuadro que contienen los triángulos, además implementó una unidad de área errada, ya que en la actividad se estableció como unidad cuadros de un centímetro cuadrado (ver Figura 6).

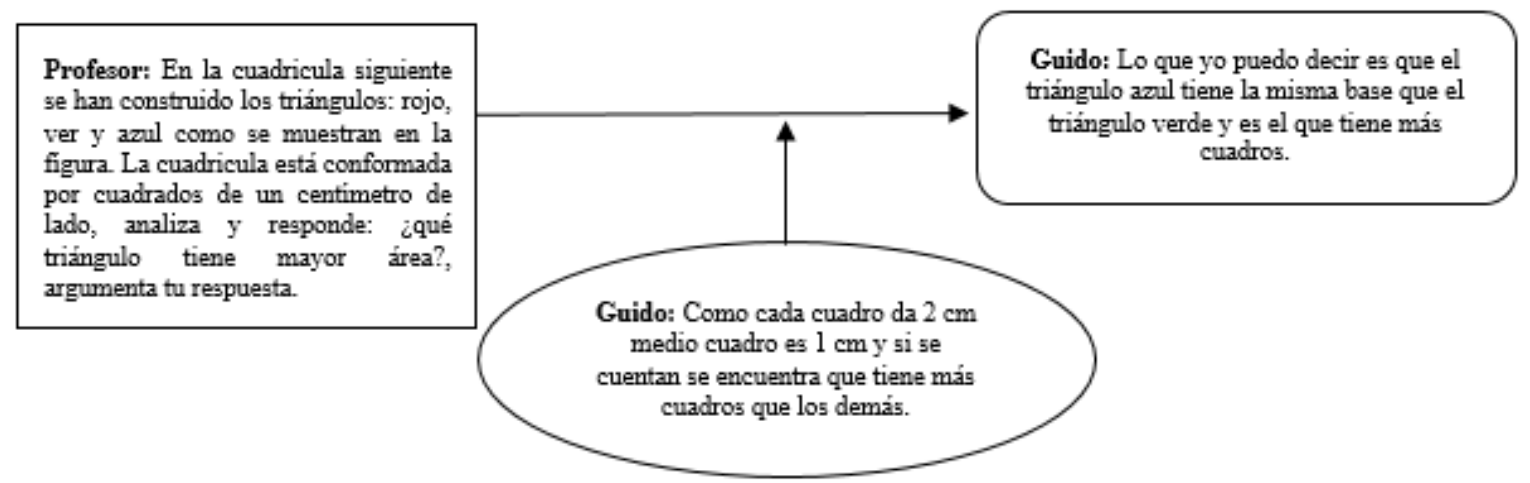

Figura 6 - Argumento de Guido Fuente: Autores

Luego de que los estudiantes comentaran sus respuestas, el estudiante Miguel emitió su aserción: el triángulo rojo tiene más área, y la garantía de su argumento se centra en contar los cuadros dentro del triángulo rojo, valido, pero no formal para el nivel escolar. El hecho de comparar la cantidad de cuadros que contiene el triángulo rojo con la cantidad de los demás triangulos dislumbra la manera de calcular el área de éstos. Ante esta aserción, se identifica la primera refutación en la clase, donde el estudiante Eduardo refutó la aserción de Miguel, y presentó su garantía con el mismo sentido del argumento de Miguel pero con datos adecuados que hacen alusión al conteo (Figura 7).

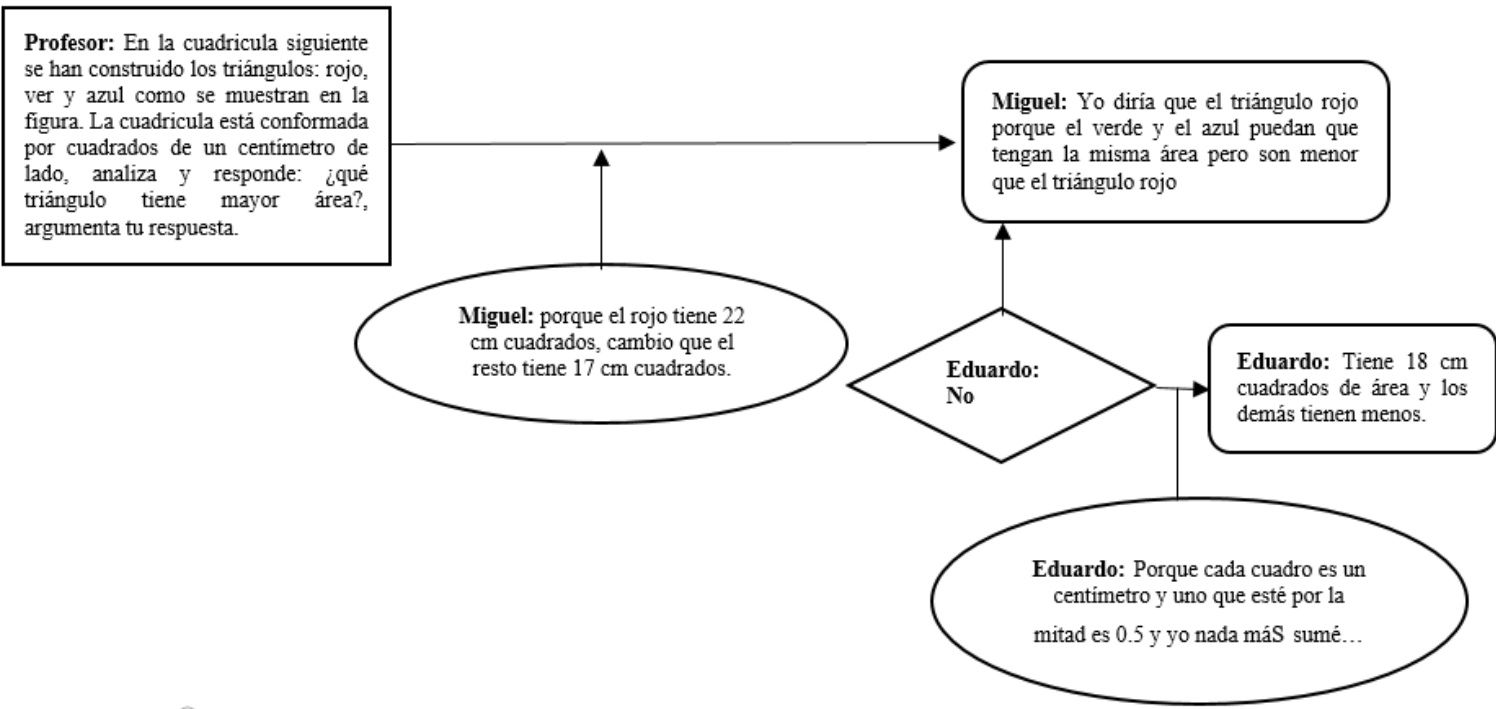

Figura 7- Argumento de Miguel y Eduardo

Fuente: Autores 
La garantía expuesta por el estudiante Eduardo especifica un dato que no tuvo en cuenta Miguel, éste propuso garantía adecuada, pero no la válida, para conectar los datos y la aserción del argumento, debido que, en la representación gráfica de la actividad matemática no todos los cuadros que rodean los lados del triángulo están divididos justamente por la mitad, garantía sustentada en lo visual, por ende, este argumento es catalogado como informal.

El estudiante Andrés manifestó su aserción; Yo digo que sería el triángulo verde y presentó en su garantía características visuales de los triángulos, además utiliza términos como; Yo digo que..., entonces para mí sería... los cuales manifiestan la ausencia de sustento matemático en la garantía y permite identificar que el argumento de este estudiante es de tipo informal.

De forma seguida, intervino un estudiante que refutó la aserción de Andrés y los demás compañeros Pues... voy a decir que: jestá mal!, presentó la aserción en total que todos los triángulos nos dan la misma cantidad... Esta aserción se fundamenta en una garantía formal, debido a que identificó la medida de las bases y sus respectivas alturas, para luego recurrir la fórmula del área del triángulo. Se puede concluir que este argumento es válido (ver Figura 8). 


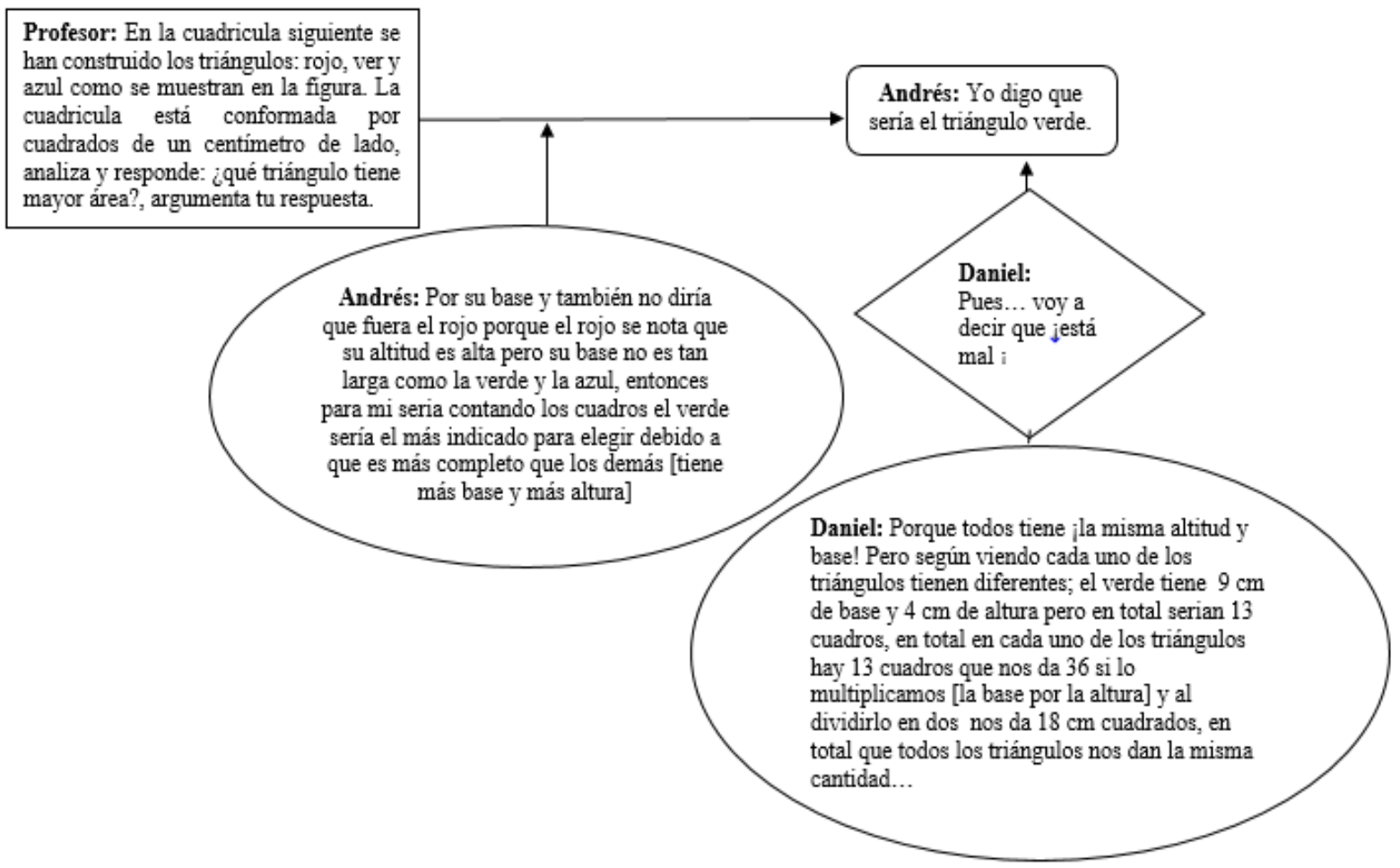

Figura 8 - Argumento de Andrés y Daniel Fuente: Autores

A partir de la refutación realizada por el estudiante Daniel, se evidenció cómo los demás estudiantes, que habían dado respuestas distintas a esta última, cambiaron sus argumentos de acuerdo al argumento presentado por Daniel. Ángel, quien había establecido su argumento en (Figura 5), ahora presenta un argumento basado en las garantías expuestas por Daniel, este cambio de argumentos fue originado por la refutación realizada por el estudiante Daniel (Figura 9).

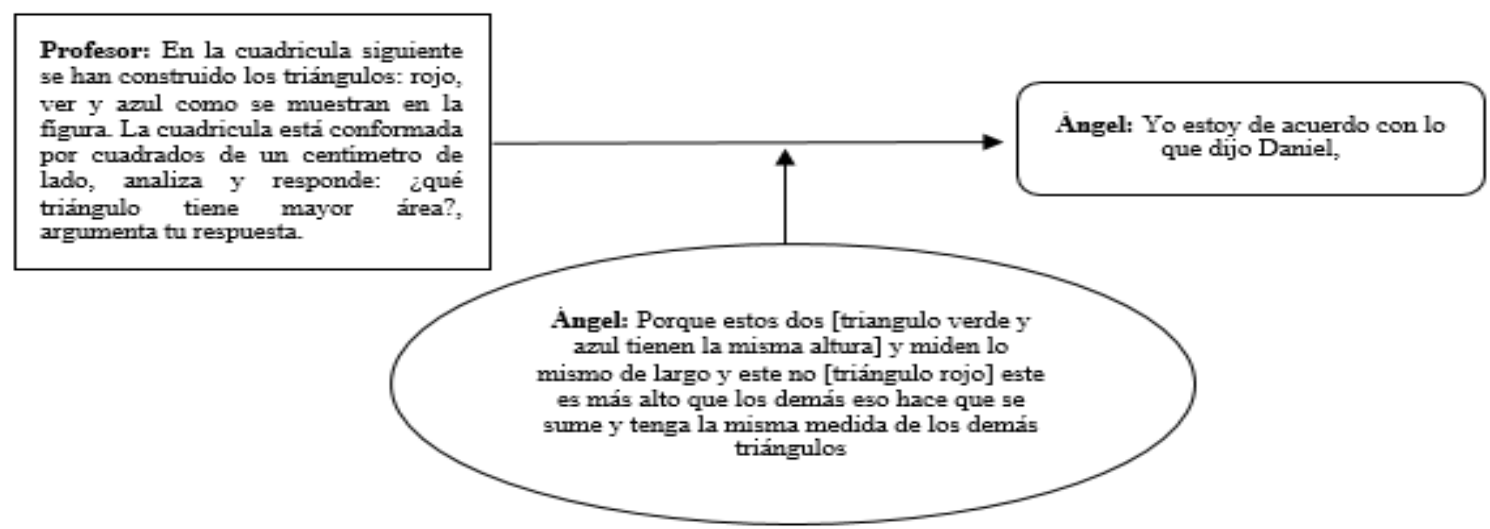

Figura 9 - Nuevo argumento de Ángel Fuente: Autores

De igual forma, intervino Guido; presentó su nuevo argumento también basado en las garantías expuestas por Daniel, y concluyó que todos tienen la misma área (Figura 10). 


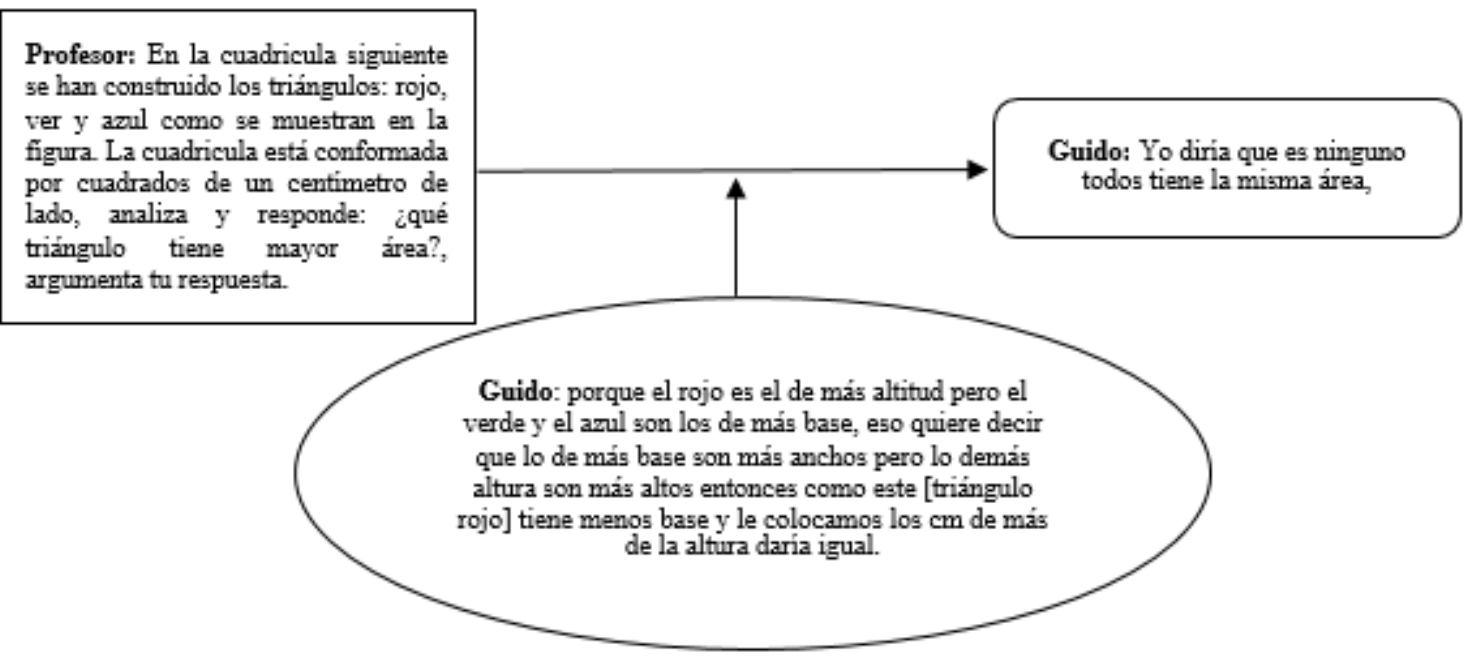

Figura 10 - Nuevo argumento de Guido Fuente: Autores

Por último, Miguel manifestó que está de acuerdo con el argumento presentado por Daniel, cambiando su respuesta; en este caso, no hace explícito cual es su argumento, pero al parecer es totalmente lo mismo que expuso Daniel (Figura 11).

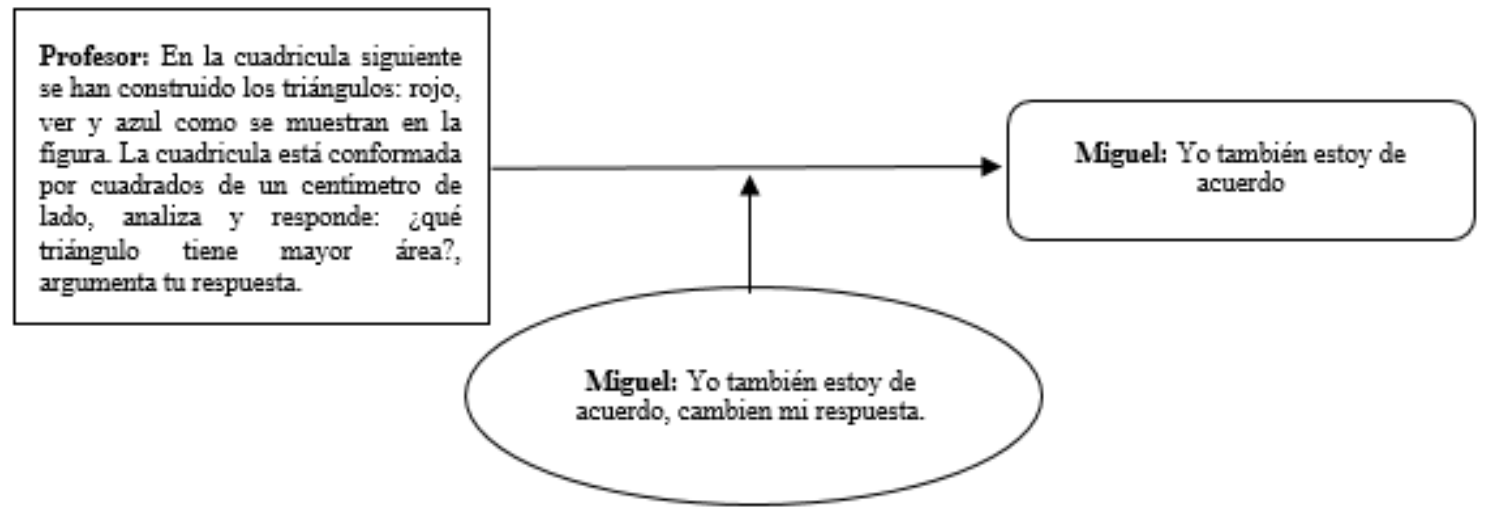

Figura 11 - Nuevo argumento de Miguel Fuente: Autores

\subsection{Refutación y la suficiencia de la garantía}

En este apartado, la refutación hace evidente la suficiencia de las garantías expuestas en los argumentos de los estudiantes (REID; KNIPPING; CROSBY, 2011). Particularmente, en el caso del argumento de Miguel y Eduardo (Figura 12), dado que el estudiante Eduardo refutó la aserción de Miguel permitió evidenciar la insuficiencia de la garantía expuesta por éste; debido a que no se corresponde la cantidad de cuadros establecidos por Miguel con la cantidad de cuadros de la figura, ésta una insuficiencia de la garantía evidenciada luego de que Eduardo refutara. 


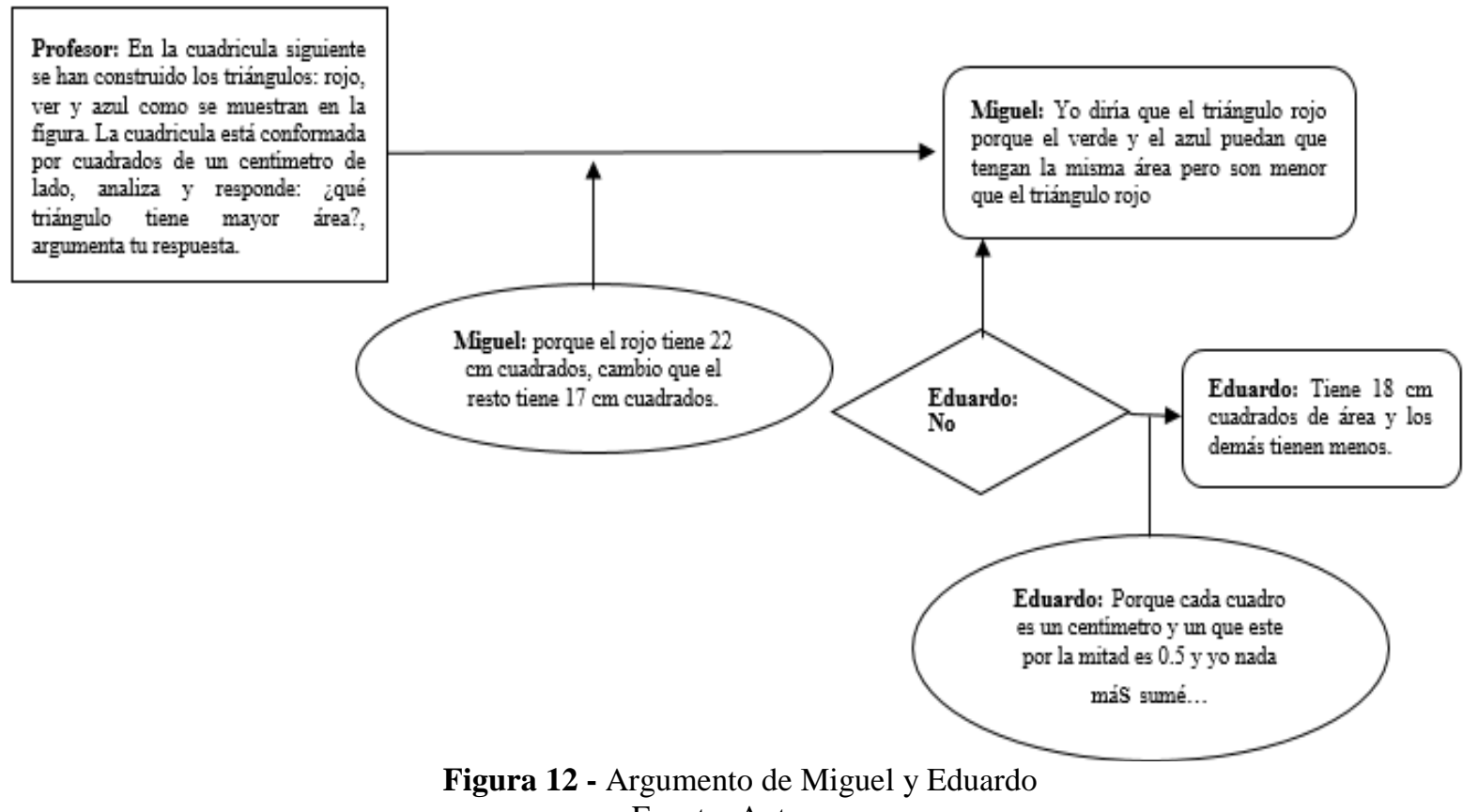

Fuente: Autores

\section{Conclusiónes}

Luego de realizar el análisis de la escena suscitada en el salón de clases, se identificaron funciones de la refutación de aserciones. La primera función es el poder persuasivo que tiene la refutación establecida por un estudiante frente una serie de argumentos ya establecidos. La refutación originó un cambio en el contenido de garantías y aserciones de los argumentos emitidos por los demás, esto siempre y cuando la refutación presente una garantía formal. Además, una segunda función de la refutación es evidenciar la insuficiencia de las garantías expuestas en los argumentos de los estudiantes. Esto a través de la presentación de una refutación que vaya dirigida a la aserción, ésta una oportunidad para mostrar que tan fuerte es la garantía del argumento expuesto por el argumentador. Por último, cabe resaltar que el modelo argumentativo de Toulmin, y la convención realizada sobre éste, facilitaron el análisis estructural de los argumentos, permitiendo que la refutación fuera identificada y sobre todo, evidenciar los aspectos importantes de la refutación de argumentos.

\section{Referencias}

BALACHEFF, N. Treatment of refutations: Aspects of the complexity of a constructivist approach to mathematics learning. En $E$. von Glasersfeld (ed.). Radical Constructivism in Mathematics Education: Netherlands, v.7, 1991. p. 89-110.

BIKNER-AHSBAHS, A.; KNIPPING, C.; PRESMEG, N. Approaches to Qualitative Research 
in Mathematics Education: Examples of methodology and methods. Research in mathematics education Aust-Adger. v. 17, n. 3, p. 247-256, Nov. 2015.

CONNER, A.; SINGLETARY, L.; SMITH, R.: WAGNER, P.; FRANCISCO, R. Teacher support for collective argumentation: A framework for examining how teachers support students' engagement in mathematical activities, Educational Studies Mathematics, v. 86 n. 2, p. 401-429, Abril 2014.

FUJITA, T. KEITH. J.; SUSUMU. K.; HIROYUKI, K.; SHINICHIRO, M. Proofs and Refutations in Lower Secondary School Geometry. In: CERME 7. 2011, Rzeszów. Proceedings of CERME 7, Rzeszów: Pytlak, 2011. p. 660-669.

GIANNAKOULIAS, E.; MASTORIDES, E.: POTARI, D.; ZACHARIADES, T. Studying teachers' mathematical argumentation in the context of refuting students' invalid claims. Journal for mathematical Behavior, Orlando, v. 3, n. 29, p. 160-168, Sep. 2010.

GOIZUETA, M.; PLANAS, N. Temas emergentes del análisis de interpretaciones del profesorado sobre la argumentación en clase de matemáticas. Enseñanza de la ciencia. v.31, n.1, p. 61-78, 2013.

HAREL, G. 'Students' proof schemes revisited: historical and epistemological considerations'. In: P. Boero (ed.): Theorems in school: From history, epistemology and cognition to classroom practice. Rotterdam: Sense, 2007. p. 65-78.

HERBST, P. G. Teaching geometry with problems : Negotiating instructional situations and mathematical tasks. Journal for Research in Mathematics Education, Usa, v. 4, n. 37, p. 313-347, 2006. Disponible en: <http://doi.org/10.2307/30034853>. Acceso en: May. 2015.

INGLIS, M.; MEJÍA-RAMOS, J. P. On the persuasiveness of visual arguments in mathematics.

Foundations of Science, Holanda, v. 2, n. 14, p. 97-110, Nov. 2008. Disponible en:

<http://doi.org/10.1007/s10699-008-9149-4>. Acceso en: 12 sep. 2015.

INGLIS, M.; MEJIA-RAMOS, J. P. La fuerza de la aserción y el poder persuasivo en la argumentación en matemáticas. Revista EMA: Investigación e Innovación en Educación Matemática, Ciudad de México, v. 3, n. 10, p. 327-352, Feb. 2005.

INGLIS, M.; MEJIA-RAMOS, J. P.; SIMPSON, A. Modelling mathematical argumentation: The importance of qualification. Educational Studies in Mathematics, Holanda, v. 1, n. 66, p. 3-21, Sep.2007. Disponible en: 〈http://doi.org/10.1007/s10649-006-9059-8>. Acceso en: 13 Sep. 2015

KRUMMHEUER, G. The ethnology of argumentation. In: COBB, P; BAUERSFELD, H. (Ed.). The Emergence of Mathematical Meaning: Interaction in Classroom Cultures. Hillsdale: Erlbaum, 1995. p. 229-269.

KRUMMHEUER, G. Argumentation and participation in the primary mathematics classroom. Two episodes and related theoretical abductions. Journal of Mathematical Behavior. Amsterdam, v. 1, n. 26, p. 60-82, April 2007.

KRUMMHEUER, G. The relationship between diagrammatic argumentation and narrative argumentation in the context of the development of mathematical thinking in the early years. Educational Studies in Mathematics, Holanda, v. 2, n. 84, p. 249-265, Octubre 2013. Disponible en: <https://link.springer.com/article/10.1007/s10649-013-9471-910649>. Acceso en: 12 Marzo. 2013.

KRUMMHEUER, G. Methods for Reconstructing Processes of Argumentation and Participation in Primary Mathematics Classroom Interaction. In A. Bikner-Ahsbahs.; C. Knipping.; N. Presmeg (Eds.). Approaches to Qualitative Research in Mathematics Education: Examples of methodology, 2015. P. 51-74. 
LAKATOS, I. Pruebas y refutaciones: La lógica del descubrimiento matemático (trad. C. Solis). Madrid: Alianza, 1986.

NATIONAL COUNCIL OF TEACHERS OF MATHEMATICS. Principles and standards for school mathematics. National Council of Teachers of Mathematics (Ed.). Usa, Nov. 2000.

REID, D. Conjectures and Refutations in Grade 5 Mathematics. Journal for Research in Mathematics Education, Michigan, v. 1, n. 33, p. 5-29, Ene. 2002.

REID, D.; KNIPPING, C.; CROSBY, M. Refutations and the logic of practice. PNA, España, v. 6, n. 1, p. 1-10, jul 2011.

TOULMIN, S. The uses of argument. (2 ed.) Cambridge: Cambridge University Press, 2003.

TOULMIN, S.; RIKIE, R.; JANIK, A. An introduction to Reasoning. Collier Maemillan. New York: Maemillan Publishing Company, 1984. Edición temprana.

VIHOLAINEN, A. Prospective mathematics teachers' informal and formal reasoning about the concepts of derivative and differentiability. 2008. 81f. Tesis (Doctorado en Educación Matemática) -Departamento de Matemáticas y Estadística, Universidad de Jyv“askyl”a, jul. 2008.

WAGNER, P; SMITH, R.; CONNER, A.; SINGLETARY, M.; FRANCISCO, R. Using Toulmin's Model to Develop Prospective Secondary Mathematics Teachers' Conceptions of

Collective Argumentation. Mathematics Teacher Educator, Usa, v. 3, n. 1, p. 8-26, Sep. 2014.

WALTON, D. Objections, Rebuttals and Refutations. 2009. Disponible en <http://scholar.uwindsor .ca/ossaarchive/OSSA8/papersandc ommentaries/151>. Acceso en: 29 mayo. 2015.

WHITENACK, J. W.; KNIPPING, N. Argumentation, instructional design theory and students' mathematical learning: A case for coordinating interpretive lenses. Journal of Mathematical Behavior, Usa, v. 4, n. 21, p. 441-457, Nov. 2002.

YACKEL, E. What we can learn from analyzing the teacher's role in collective argumentation. Journal of Mathematical Behavior, Usa, v. 4, n. 21, p. 423-440, Nov. 2002.

YOPP, D. Prospective elementary teachers' claiming in responses to false generalizations. Journal of Mathematical Behavior, Usa, v. 39, n.1, p. 79-99, Jun. 2015.

Submetido em 30 de Junho de 2016. Aprovado em 7 de Março de 2017. 
Anexos

\begin{tabular}{|c|c|c|}
\hline escena & Participante & dialogo \\
\hline 1. & Profesor & $\begin{array}{l}\text { En la cuadrícula siguiente se han construido los triángulos: rojo, ver y azul como } \\
\text { se muestran en la figura. La cuadricula está conformada por cuadrados de un } \\
\text { centímetro de lado, analiza y responde: ¿qué triángulo tiene mayor área?, } \\
\text { argumenta tu respuesta. } \\
\text { Vamos a escuchar las respuestas de ustedes: }\end{array}$ \\
\hline 2. & Nicolás & $\begin{array}{l}\text { Es el área del triángulo verde, porque se ve que tiene más latitud lo cual es altura, } \\
\text { lo cual lleva a cabo más cuadritos (serian 14). También porque el rojo tiene más } \\
\text { longitud (base) pero no la suficiente altura, y el azul tiene más altura pero no más } \\
\text { base. El verde tiene las dos [base y altura] el cual caben más centímetros. }\end{array}$ \\
\hline 3. & Profesor & ¿Qué pueden decir de lo que dijo Nicolás? \\
\hline 4. & Andrés & Estoy de acuerdo con lo que dijo Nicolás. \\
\hline 5. & Ángel & $\begin{array}{l}\text { Yo creo que el que tiene más área es el azul[ triángulo azul] porque contando los } \\
\text { cuadros el azul tiene más cuadros y debido a estos la medida del área es mayor, }\end{array}$ \\
\hline 6. & Profesor & $\begin{array}{l}\text { Él cuenta los cuadros y observa que tiene más cuadros que los demás triángulos, } \\
\text { ¿qué pueden decir de su respuesta? }\end{array}$ \\
\hline 7. & Guido & $\begin{array}{l}\text { Lo que yo puedo decir es que el triángulo azul tiene la misma base que el triángulo } \\
\text { verde y es el que tiene más cuadros y como cada cuadro da } 2 \mathrm{~cm} \text {, medio cuadro es } \\
1 \mathrm{~cm} \text { y si se cuentan se encuentra que tiene más cuadros que los demás. }\end{array}$ \\
\hline 8. & Profesor & Mi amigo miguel, cuéntame... \\
\hline 9. & Miguel & $\begin{array}{l}\text { Yo diría que el triángulo rojo porque el verde y el azul puedan que tengan la } \\
\text { misma área pero son menor que el triángulo rojo, porque el rojo tiene } 22 \mathrm{~cm} \\
\text { cuadrados, cambio que el resto tiene } 17 \mathrm{~cm} \text { cuadrados. }\end{array}$ \\
\hline 10. & Profesor & ¿Y cómo sabes que tiene $17 \mathrm{~cm}$ cuadrados? \\
\hline 11. & Miguel & Porque yo había hecho las operaciones \\
\hline 12. & Profesor & ¿Qué dicen de lo que dijo Miguel? \\
\hline 13. & Profesor & Mi amigo Eduardo, ¿qué dices? \\
\hline 14. & Eduardo & No, Tiene $18 \mathrm{~cm}$ cuadrados de área y los demás tienen menos. \\
\hline 15. & Profesor & ¿Cómo sabes que tienes $18 ?$ \\
\hline 16. & Eduardo & $\begin{array}{l}\text { Porque cada cuadro es un centímetro y un que este por la mitad es } 0.5 \text { y yo nada } \\
\text { más sumé... }\end{array}$ \\
\hline 17. & Miguel & $\begin{array}{l}\text { No, contaron mal porque si hacemos el área total de un cuadrado de lado } 1 \\
\text { centímetro daría } 2 \mathrm{~cm} \text { cuadrados }\end{array}$ \\
\hline 18. & Profesor & ¿Por qué? \\
\hline 19. & Miguel & $\begin{array}{l}\text { Multiplique } 1 \text { x } 1 \text { y esto de me daría } 1 \text { y si dividimos por } 2 \text { me daría dos } \\
\text { centímetro cuadrados }\end{array}$ \\
\hline 20. & Profesor & ¿Si lo divides por dos?, No te da 0.5 \\
\hline 21. & Miguel & Ahhhh si daría $0.5 \ldots$ \\
\hline 22. & Profesor & Vamos a escuchar a mi amigo Andrés \\
\hline 23. & Andrés & $\begin{array}{l}\text { Yo digo que sería el triángulo verde, por su base y también no diría que fuera el } \\
\text { rojo porque el rojo se nota que su altitud es alta pero su base no es tan larga como } \\
\text { la verde y la azul, entonces para mi seria contando los cuadros el verde sería el } \\
\text { más indicado para elegir debido a que es más completo que los demás [ tiene más } \\
\text { base y más altura] }\end{array}$ \\
\hline 24. & Profesor & ¿Qué pueden decir de lo que dijo mi amigo Andrés? \\
\hline 25. & Daniel & Pues... voy a decir que ¡está mal ¡ \\
\hline 26. & Profesor & ¿Por qué está mal? \\
\hline 27. & Daniel & $\begin{array}{l}\text { Porque todos tienen ¡la misma altitud y base! } \\
\text { Pero según viendo cada uno de los triángulos tienen diferentes; el verde tiene } 9 \mathrm{~cm} \\
\text { de base y } 4 \mathrm{~cm} \text { de altura pero en total serian } 13 \text { cuadros, en total en cada uno de } \\
\text { los triángulos hay } 13 \text { cuadros que nos da } 36 \text { si lo multiplicamos [la base por la } \\
\text { altura] y al dividirlo en dos nos da } 18 \mathrm{~cm} \text { cuadrados, en total que todos los } \\
\text { triángulos nos dan la misma cantidad... }\end{array}$ \\
\hline 28. & Profesor & ¿Qué pueden decir de lo que dijo mi amigo Daniel? \\
\hline 29. & Ángel & Yo estoy de acuerdo con lo que dijo Daniel, porque estos dos [ triangulo verde y \\
\hline
\end{tabular}




\begin{tabular}{|c|l|l|}
\hline & & $\begin{array}{l}\text { azul tienen la misma altura] y miden lo mismo de largo y éste no [ triángulo rojo] } \\
\text { éste es más alto que los demás eso hace que se sumen y tenga la misma medida de } \\
\text { los demás triángulos }\end{array}$ \\
\hline 30. & Profesor & Pero tú dijiste primero que era el triángulo azul i \\
\hline 31. & Ángel & Si pero ya me di cuenta que es incorrecto \\
\hline 32. & Guido & $\begin{array}{l}\text { Yo diría que es ninguno, todos tienen la misma área, porque el rojo es el de más } \\
\text { altitud pero el verde y el azul son los de más base, eso quiere decir que lo de más } \\
\text { base son más anchos pero lo demás altura son más altos entonces como este } \\
\text { [triángulo rojo] tiene menos base y le colocamos los cm de más de la altura daría } \\
\text { igual. }\end{array}$ \\
\hline 33. & Miguel & Yo también estoy de acuerdo, cambien mi respuesta. \\
\hline 34. & Profesor & iY por qué dijiste primero que era el triángulo rojo? \\
\hline 35. & Miguel & Porque medí mal.... \\
\hline
\end{tabular}

Cuadro 1 Transcripción de la escena. 